PHYSICAL REVIEW D 95, 109904(E) (2017)

\title{
Erratum: Propagation of test particles and scalar fields on a class of wormhole space-times [Phys. Rev. D 90, 024057 (2014)]
}

Peter Taylor

(Received 3 May 2017; published 25 May 2017)

DOI: 10.1103/PhysRevD.95.109904

In Sec. III of our paper, we considered the stability of solutions of the scalar wave equation on a class of wormhole backgrounds. Whether or not the solutions are stable depends on the value of the coupling of the scalar to the background curvature. We showed that these regions of instability are related to an infinite set of spurious poles in the expression for the self-force on a scalar charge held static in these wormhole spacetimes. Specifically, all the values of the coupling constant for which the self-force diverges lie outside the domain of stability of the scalar wave equation. By restricting our study to the stable domain of solutions, we obtain sensible (finite) results for the self-force. Despite an error, which we correct here, propagating throughout Sec. III of that paper, these conclusions are robust. Indeed, the explanation for the spurious poles in the self-force as an artifact of considering scalar field solutions in the unstable domain remains the correct one. Nevertheless, we correct the error in that section, explaining the main formulas which it affects.

As a result of notational inconsistency in the metric functions, in particular, in what was called the shape function $b(r)$ for the wormhole throat, there was an error in the expression for the background curvature scalar given in Eq. (3.2). Instead, the Ricci scalar $R$ for arbitrary shape function $b(r)$ should have read

$$
R=\frac{2 b^{\prime}(r)}{r^{2}}
$$

This in turn affects the scalar wave equation (3.3). After a separation of variables, the radial equation (3.5) should have read

$$
\left\{\left(1-\frac{b(r)}{r}\right)^{1 / 2} \frac{d}{d r}\left(\left(1-\frac{b(r)}{r}\right)^{1 / 2} \frac{d}{d r}\right)+\omega^{2}-\frac{l(l+1)}{r^{2}}-2\left(\xi-\frac{1}{4}\right) \frac{b^{\prime}(r)}{r^{2}}-\frac{b(r)}{2 r^{3}}\right\} \chi_{\omega l}(r)=0 .
$$

Then after rewriting in terms of proper radial distance $\rho$, the potential in Eq. (3.7) should instead be

$$
V_{l}(\xi, \rho)=\frac{l(l+1)}{r^{2}}+2\left(\xi-\frac{1}{4}\right) \frac{b^{\prime}(r)}{r^{2}}+\frac{b(r)}{2 r^{3}} .
$$

If we now consider the particular class of wormholes defined by the shape functions $b(r)=b_{0}^{1-q} r^{q}$ for $q<1$, then the Ricci scalar in Eq. (3.8) should be

$$
R=\frac{2 q}{r^{2}}\left(\frac{b_{0}}{r}\right)^{1-q}
$$

and the potential simplifies to

$$
V_{l}(\xi, \rho)=\frac{1}{r^{2}}\left(l(l+1)+\left(\frac{b_{0}}{r}\right)^{1-q}\left\{2 q \xi+\frac{1}{2}(1-q)\right\}\right) .
$$

From this potential, we can deduce that the scalar field $\varphi$ is stable whenever $2 q \xi+\frac{1}{2}(1-q)>0$. Solving this inequality for $\xi$ implies that

$$
\xi>\frac{q-1}{4 q} \text { if } 0<q<1, \quad \xi<\frac{q-1}{4 q} \text { if } q<0 .
$$

These inequalities imply, for example, that all scalar solutions, regardless of shape exponent $q<1$, are stable if the coupling constant is in the range $0 \leq \xi \leq \frac{1}{4}$. We also note that for $q=0$, the Ricci scalar vanishes and hence $\xi$ does not appear in the 
potential. In this case, the scalar field solutions are stable since the potential is clearly positive definite. Another popular choice of shape exponent is $q=-1$, which corresponds to the so-called Ellis wormhole. Stable solutions on this background are restricted to couplings in the range $\xi<1 / 2$. In general, we see that the stability of solutions corresponding to the corrected potential (5) is richer than that of the erroneous potential in our paper where we had stable solutions for $\xi<1 / 2$ independent of shape exponent $q$.

In Sec. III B, we computed reflection and transmission coefficients in a fourth-order WKB approximation. All the results in this section are given in terms of the potential and its derivatives evaluated at $\rho=0$. Hence the explicit forms of all the equations (3.13)-(3.19) remain valid with the understanding that the potential and its derivatives should be evaluated using (5) above.

In Sec. III C, we further investigated the stability of scalar solutions by computing the quasinormal mode (QNM) frequencies, again adopting the fourth-order WKB method. The square of the QNM frequencies is given by Eq. (3.20), and again the formula remains valid provided we replace the potential and its derivatives with (5) above. An explicit expression (3.21) for the QNMs was given in the large $l$ limit and this should instead read

$$
\begin{aligned}
\operatorname{Re}(\omega)= & \frac{1}{b_{0}}\left(l+\frac{1}{2}\right)-\frac{1}{128 b_{0}\left(l+\frac{1}{2}\right)}\left[16-128 \xi+(1-q)(128 \xi-22)+(1-q)^{2}+4\left(n+\frac{1}{2}\right)^{2}(1-q)(3-q)\right]+\mathcal{O}\left(l^{-3}\right), \\
\operatorname{Im}(\omega)= & -\frac{\left(n+\frac{1}{2}\right) \sqrt{1-q}}{\sqrt{2} b_{0}}\left\{1-\frac{(1-q)}{6144 b_{0}\left(l+\frac{1}{2}\right)^{2}}\left[380-3072 \xi+12(1-q)(256 \xi-43)+31(1-q)^{2}\right.\right. \\
& \left.\left.-4\left(n+\frac{1}{2}\right)^{2}(5 q+17)(3-q)\right]\right\}+\mathcal{O}\left(l^{-3}\right) .
\end{aligned}
$$

Finally in Sec. III C, we give a simple alternative approximation for computing the QNM frequencies by fitting the inverted potential to the Pöschl-Teller potential and relating the QNMs to the known bound states of this potential. Assuming that our potential is positive for all $l$ (which implies stable solutions) and replacing (3.22) in our paper with the following definitions

$$
\lambda^{2}=l(l+1), \quad \mu^{2}=2 q \xi+\frac{1}{2}(1-q),
$$

then the potential in (3.23) should be written as

$$
V=\frac{\lambda^{2}}{r^{2}}+\frac{\mu^{2}}{r^{2}}\left(\frac{b_{0}}{r}\right)^{1-q} .
$$

A good approximation to this potential is given by the Pöschl-Teller potential

$$
V_{\mathrm{PT}}=\frac{V_{0}}{\cosh ^{2}(\alpha \rho)},
$$

where instead of (3.26), we should have

$$
V_{0}=\frac{\lambda^{2}+\mu^{2}}{b_{0}^{2}}, \quad \alpha=\frac{1}{2 b_{0}} \sqrt{\frac{(1-q)\left(2 \lambda^{2}-\mu^{2}(q-3)\right)}{\lambda^{2}+\mu^{2}}} .
$$

The bound states of this potential are well known and can be used to give an approximation for the QNMs. The result is that (3.28) should be replaced by

$$
\begin{aligned}
\omega= & \left(\frac{l(l+1)+2 q \xi+\frac{1}{2}(1-q)}{b_{0}^{2}}-\frac{(1-q)(4 l(l+1)-(q-3)(1-q+4 q \xi))}{16 b_{0}^{2}(2 l(l+1)+1-q+4 q \xi)}\right)^{1 / 2} \\
& -i\left(n+\frac{1}{2}\right)\left(\frac{(1-q)(4 l(l+1)-(q-3)(1-q+4 q \xi))}{4 b_{0}^{2}(2 l(l+1)+1-q+4 q \xi)}\right)^{1 / 2} .
\end{aligned}
$$

The main point is that $\operatorname{Im}(\omega)<0$ which implies stability of the scalar solutions. This procedure is straightforward to adapt to compute the modes corresponding to a negative definite potential with a single minimum. For example, for the $l=0$ 
mode, this requires $4 q \xi+1-q<0$. In this case, in order to cast it in the form of a bound-state problem, we require a set of transformations that reverse the sign of the potential; the appropriate choice is

$$
\rho \rightarrow-i \rho, \quad b_{0} \rightarrow-i b_{0} .
$$

Following the same procedure as in our paper, we obtain after analytically continuing back

$$
\omega=i \alpha\left(n+\frac{1}{2}\right)+\left(-\frac{1}{4} \alpha^{2}-V_{0}\right)^{1 / 2},
$$

where $\alpha$ and $V_{0}$ are given by (11) above (with $V_{0}<0$ ). In this case, we have $\operatorname{Im}(\omega)>0$ giving rise to an unstable mode. 